\title{
Hors-thèmes : La mémoire des survivants et la révolte des ombres : les disparus dans la société chilienne (1973-1995)
}

\section{Antonia GARCIA CASTRO}

\section{CpenEdition}

\section{Journals}

Édition électronique

URL : http://journals.openedition.org/conflits/2163

DOI : $10.4000 /$ conflits. 2163

ISSN : $1777-5345$

Éditeur :

CCLS - Centre d'études sur les conflits lilberté et sécurité, L'Harmattan

Édition imprimée

Date de publication : 15 mai 1997

ISSN : 1157-996X

Référence électronique

Antonia GARCIA CASTRO, « Hors-thèmes : La mémoire des survivants et la révolte des ombres : les disparus dans la société chilienne (1973-1995) », Cultures \& Conflits [En ligne], 24-25 | hiver 1996printemps 1997, mis en ligne le 27 mars 2007, consulté le 30 mars 2021. URL : http:// journals.openedition.org/conflits/2163; DOI : https://doi.org/10.4000/conflits.2163

Ce document a été généré automatiquement le 30 mars 2021

Creative Commons License 


\title{
Hors-thèmes : La mémoire des survivants et la révolte des ombres : les disparus dans la société chilienne (1973-1995)
}

\author{
Antonia GARCIA CASTRO
}

1 S'il fallait fixer une date marquant le début du processus de démocratisation au Chili, peut-être faudrait-il opter pour ce 5 octobre 1988, où le peuple chilien a été invité à se prononcer sur l'avenir du régime militaire. Ce choix serait moins motivé par l'issue de ce référendum ${ }^{1}$ que par sa mise en œuvre. En effet, pour la première fois depuis le coup d'Etat de 1973, deux conceptions, non pas seulement de l'avenir, mais aussi du passé du Chili, se sont opposées ouvertement. Poser la question de la capacité des militaires à gouverner le pays ne pouvait que solliciter une réflexion plus ample sur la manière dont le pays avait été, effectivement, gouverné par les militaires. Cette présence du passé devint, durant les années quatre-vingt-dix, un thème majeur du débat politique, soutenu par l'interrogation suivante : " Que faut-il connaître des pratiques répressives du régime militaire afin de consolider la transition démocratique ? ". Le fait que la transition ait été une initiative du régime militaire lui-même place le nouveau gouvernement dans une situation complexe : limité dans son action par des entraves institutionnelles qui protègent les intérêts des forces armées², il ne peut cependant ignorer les revendications des secteurs politiques dont il est issu. Or, la thématique des violations des droits de l'homme, perpétrées sous le régime militaire, a été le ferment de la coalition qui remporta les élections du 14 décembre $1989^{3}$.

2 Le crime de la disparition-forcée est au cœur de ce débat qui cherche à établir ce qu'il faut retenir des événements de 1973. Il en est ainsi, parce que son existence donna lieu à une organisation qui, dès 1975, s'opposa publiquement au régime : l'Association des Familles des Détenus-Disparus (A.F.D.D.). Ce sont les mères des disparus qui, les premières, sont descendues dans les rues de Santiago pour demander aux autorités compétentes où se trouvaient leurs enfants. Leur prise de parole fraya un chemin de 
liberté qui fut progressivement emprunté par d'autres ${ }^{4}$. La lutte des familles des disparus devint ainsi un des symboles de la résistance au régime militaire. Mais surtout, la manière dont ces femmes entrèrent dans la vie politique chilienne, modifia sensiblement la nature du conflit qui opposait les partisans de l'ancien gouvernement socialiste aux Forces Armées : c'est au grand jour et sans armes qu'elles dressèrent, à la vue de tous, les images de la disparition-forcée déjouant ainsi la stratégie d'invisibilisation du pouvoir ${ }^{5}$.

3 Or, si le crime de la disparition-forcée pose actuellement un problème au gouvernement démocratique, c'est moins parce qu'il est un objet du débat que parce qu'il interroge la société chilienne sur la légitimité même de ceux qui prennent la parole : comment juger aujourd'hui le crime de la disparition-forcée sans porter un jugement sur la nature du pouvoir alors en place ? Comment prononcer un jugement si l'on ne dispose pas, par ailleurs, du pouvoir de le faire ? La manière dont le débat est posé importe peut-être plus que l'issue du débat lui-même car les mots prononcés seront, quoi qu'il advienne, légués aux prochaines générations. Et cette certitude détermine aussi la manière dont se joue le rapport de force opposant ceux qui gouvernent et ceux qui ont gouverné. Ainsi, la question initiale importe peu si elle n'est pas accompagnée de cette autre question : " Comment ces prochaines générations, se souviendront-elles des événements de 1973 ? Comment se souviendront-elles de ces hommes et de ces femmes qui en furent les acteurs ? ". C'est là, peut-être, une des principales victoires des familles des détenus-disparus : avoir fait de la disparitionforcée un miroir où chacun peut se regarder tel qu'il sera aux yeux des générations à venir.

Les paradoxes de la disparition

$4 \quad$ S'il n'est pas aisé de concevoir le crime de la disparition-forcée, c'est peut-être parce que sa principale caractéristique est une absence. Dire d'une personne qu'elle " a disparu " ne nous renseigne nullement sur son sort : elle peut être morte, elle peut être vivante, elle est considérée comme " disparue " parce qu'un jour elle n'a plus été " vue " par son entourage. La disparition-forcée suppose toujours l'occultation du corps de la victime. Et tant que le corps n'est pas visible, tant qu'il demeure introuvable, quiconque pratique la disparition n'a pas à répondre de ses actes. Ainsi, c'est l'existence d'un entourage qui donne un sens à l'utilisation de cette pratique. C'est parce que quelqu'un va constater la disparition d'un individu, sans pouvoir pourtant la prouver, que la disparition-forcée peut devenir un instrument du pouvoir. Entre 1973 et 1990, elle permit au régime militaire chilien d'exercer un pouvoir coercitif, sur certains secteurs de la population, tout en se défendant publiquement de porter atteinte aux droits de l'homme'.

Qui sont les disparus ? Pour la plupart, il s'agit d'hommes et de femmes appartenant à des organisations politiques (partis, syndicats, associations) ayant soutenu le gouvernement socialiste. En tant que tels, ils ont été désignés comme étant le nouvel ennemi : " La doctrine marxiste développe une conception de l'homme et de la société qui blesse la dignité humaine et porte atteinte aux valeurs chrétiennes de la tradition nationale "7. La pratique de la disparition-forcée s'inscrit dans la logique d'un discours qui cherche à déterminer quelles doivent être les valeurs de la communauté nationale, qui en fait partie et qui doit en être définitivement exclü ${ }^{8}$. Les partisans du gouvernement socialiste sont, en raison de leur appartenance politique, perçus par les nouveaux gouvernants comme étant membres d'une communauté dissidente et ils 
doivent, de ce fait, être à jamais bannis de la communauté nationale. La pratique de la disparition-forcée s'intègre donc dans ce dispositif plus large par lequel le pouvoir militaire cherche à légitimer son action et à jeter le discrédit sur ceux qu'il réprime : les militants de gauche sont de " mauvais " éléments ne pouvant que nuire, par leur simple existence, à l'ensemble du " corps " national. Les procédés de la disparition-forcée sont particulièrement " adaptés " à cette " éradication du mal " 9 . En effet, le disparu est souvent un prisonnier qui n'a pas été reconnu par les autorités militaires. Conduit dans un centre secret de détention, son corps est dérobé aux regards du monde extérieur et privé de tous ses droits. L'appropriation radicale du corps du prisonnier permet au pouvoir militaire de se prétendre absolu, en dévoilant, en même temps, l'inutilité de tout acte de résistance. Cette dimension est essentielle car l'individu qui disparaît est, aussi et surtout, un intermédiaire permettant de dissuader une collectivité donnée de s'ériger en opposition au pouvoir établi.

6 En effet, c'est l'entourage de la victime qui subit, à proprement parler, les effets de la disparition-forcée. Il y a tout lieu de croire que la disparition cherchait à atteindre, en priorité, l'entourage politique du disparu : n'importe quel moyen pouvait être utilisé pour soutirer des informations à ce prisonnier que personne n'était en mesure de défendre ${ }^{10}$; il devenait ainsi un pion permettant la capture d'autres individus qui, à leur tour, pouvaient être contraints de participer, plus ou moins directement, à des arrestations. Mais, cet entourage, organisé politiquement, n'était pas le seul visé. La disparition-forcée s'adressait également à des inconnus : quiconque était confronté à la disparition d'un proche (un parent, un ami, un collègue de travail, ou encore l'ami d'un ami...) était engagé à garder le silence car toute prise de parole pouvait conduire au " même " sort. Et c'est là, que la disparition-forcée apparaît comme une pratique coercitive tout à fait particulière, puisque personne ne savait, réellement, en quoi consistait ce sort. L'ombre qui semble ensevelir le corps du disparu affecte son entourage autrement que la certitude d'un mauvais traitement, ou même, d'une mort : c'est le doute, provoqué par la disparition d'un proche, qui constitue la blessure pour ceux qui demeurent. Mais, si n'importe quelle connaissance de la victime pouvait être intimidée par sa disparition, seul son entourage affectif pouvait éprouver cette blessure. C'est parce qu'ils avaient appris à vivre avec l'être cher, que les mères, les compagnes, les enfants des disparus vécurent désormais le moindre détail de la vie quotidienne (un anniversaire qui ne fut pas souhaité, une parole qui ne fut plus entendue) comme une anomalie insupportable.

Survivre à l'absence

7 Chez celui qui reste, la disparition provoque un sentiment de perte, vécu chaque jour avec la même intensité. Il en est ainsi, parce que le doute qui résulte de l'invisibilité du corps interdit tout rituel de la séparation : tout peut arriver à celui qui a été forcé à partir. La crainte des proches, dirige chacune des pensées vers l'absent : " Quand on m'a dit que mon frère avait été arrêté, j'ai senti que le monde s'effondrait autour de moi... Nous formions une famille très unie, mon frère, sa femme, mon mari, mon fils et moi... Nous avons passé ensemble des moments difficiles et cette unité nous donnait de la force. Alors, c'est douloureux qu'ils ne soient plus avec moi... Mais le souvenir est présent, certaines dates me les rappellent... les anniversaires de mon frère, de mon fils, de mon neveu... "11. L'expérience de la disparition se caractérise ainsi par cette " présence de l'absence ". Le disparu n'est pas un mort. Il n'y a pas de deuil permettant à son entourage d'apprendre à vivre sans lui. Dans l'incertitude de ce qui est advenu, la douleur se renouvelle chaque jour mais en même temps que l'espoir d'un retour 
prochain. Tant que cet espoir subsiste (et il subsiste tant que le corps n'a pas été retrouvé), la séparation ne peut être acceptée. Le souvenir du disparu s'impose et de même la nécessité de le chercher. La disparition-forcée constitue ainsi une expérience indépassable. Le proche du disparu " demeure ", non seulement parce qu'il est celui qui constate l'absence, mais aussi parce que cette absence agit comme une ancre qui le retiendrait dans le moment présent sans, toutefois, l'immobiliser.

8 La quête semble s'imposer, d'abord, comme le refus de vivre l'absence au quotidien : " J'ai besoin de savoir ce qui s'est passé. J'ai tellement cherché... C'est qu'on ne sait pas s'ils ont eu besoin de quelque chose... s'ils ont eu froid... s'ils ont voulu une cigarette... Comment peut-on vivre comme ça? A chaque fois que j'ai mangé quelque chose de bon, je me suis demandé s'il avait faim "12. Tout se passe comme si on ne pouvait se résoudre à cette présence de soi sans l'autre. Chaque geste de la vie quotidienne peut devenir une blessure car il projette le proche du disparu dans une normalité ressentie comme inacceptable. Ainsi, la disparition forcée peut également provoquer, chez celui qui reste, un sentiment de culpabilité. Culpabilité, devant l'impuissance : le fait que les disparitions soient pratiquées avec la complicité des autorités gouvernementales, laisse les familles des victimes sans aucun recours. Culpabilité aussi parce que l'on est soimême épargné par ce destin incertain que subit le disparu. La vie ne saurait reprendre son cours tant que l'autre " peut " avoir faim, ou soif, ou froid. Il s'agit d'apprendre à rester en vie sans que cela soit une trahison, et donc, sans accepter la séparation. La quête est aussi le lieu de cet apprentissage.

9 L'expérience de la disparition forcée s'inscrit dans la longue durée. En 1973, il n'y a pas, au Chili, d'antécédent de ce crime : les familles des victimes ne sont pas en mesure de distinguer la disparition de la simple arrestation. Ce n'est que lorsqu'elles entreprennent les premières recherches, lorsqu'elles sont confrontées aux dénégations des différentes autorités consultées, qu'elles soupçonnent le caractère particulier de cette pratique. Ainsi, leur quête est, en premier lieu, quête d'un prisonnier. L'affaire dite " des 119 " marque un événement dans le processus d'identification du crime : en juillet 1975, deux listes sont publiées dans la presse argentine et brésilienne, elles comportent les noms de 119 militants du MIR qui se seraient, selon les journalistes, exterminés entre eux ; des demandes d'habeas corpus avaient été présentées pour la majorité de ces personnes. Malgré les pressions des familles, soutenues par un secteur de l'Église catholique, le corps de leurs proches ne leur sera jamais rendu. La quête des familles change alors de nature : elle devient quête d'un prisonnier qui peut être mort ou vivant. Un temps de latence sépare le moment où l'arrestation se produit et le moment où les familles réalisent la nature du crime auquel elles sont confrontées. L'expérience de la disparition résulte ainsi d'une reconstruction permanente ${ }^{13}$.

10 Si la séparation ne peut être acceptée comme telle, c'est également parce qu'alors même qu'elle se produisait, les proches n'en avaient pas conscience : " Il était dix heures du soir, mais ils ont dit qu'ils n'en avaient pas pour très longtemps... une demiheure tout au plus... Je ne peux m'empêcher d'entendre sa voix qui me dit de ne pas m'inquiéter, qu'elle revient tout de suite. Du fond du cœur, je la vois... comme elle était lorsqu'elle est partie... ". C'est une mère qui s'exprime de la sorte. Elle a confiance dans les paroles des policiers qui lui assurent que sa fille sera de retour dans peu de temps. Ignorant alors les activités politiques de sa fille, militante du MIR, elle la laisse partir. Elle ne sait pas, elle ne peut pas savoir, que jamais elle ne la reverra " comme elle était ". L'image de ce moment revient, insidieuse, à mesure que son enquête l'amène à 
découvrir l'engagement politique de sa fille, les raisons de son arrestation, puis les procédés de ce que l'on appellera progressivement la " détention-disparition ". Si cette prise de conscience modifie la nature de la quête, c'est non seulement parce qu'elle introduit l'idée de la mort probable de l'être cher, mais aussi parce qu'elle conduit ses proches à s'interroger sur ce qu'il était avant de devenir un " disparu ". Celui qui reste, " marche sur les traces du disparu ", au propre et au figuré. C'est-à-dire, qu'il ne cherche pas seulement à retrouver un corps (mort ou vivant), ni même une certaine vérité (qui serait les conditions de l'emprisonnement et de la mort, si elle a eu lieu) mais aussi une certaine connaissance de l'être aimé. Retrouver un disparu, c'est également comprendre les raisons de cet engagement politique qui l'ont rendu haïssable pour le régime militaire et accepter de mettre son propre corps au service de ce projet. Ce faisant, le proche du disparu est comme détourné de sa voie propre : en se vouant à la quête exclusive de l'être aimé, il devient lui-même un disparu. Et c'est à cette seule condition qu'il peut survivre à l'absence.

La mémoire comme instrument de résistance

11 La quête des familles acquiert, à partir de 1975, une dimension collective. En effet, l'affaire des " listes des 119 " témoigne de l'inefficacité de toute procédure isolée, menée dans le cadre d'une légalité qui n'existe plus que comme fiction. Le pouvoir militaire développe sa propre version des faits et celle-ci dit à la population chilienne : " Il n'y a pas, il n'y a jamais eu, de disparus ". Ainsi, la publication des listes permet de tracer les contours de cette vérité officielle qui doit être acceptée sans réserves : elle témoigne de la volonté de faire taire les rumeurs, tout en avertissant les familles du danger qui guette quiconque osera contredire ce discours. L'affaire des " listes des 119 " se présente comme étant une réponse aux premières démarches faites par les familles ${ }^{14}$. De ce fait, elle est aussi un aveu implicite du crime commis. La manière dont les familles conçoivent l'action collective s'inscrit dans la logique même de ce crime. C'est l'invisibilité du corps qui permet aux autorités militaires de soutenir la non-existence des disparus. Il est donc, en premier lieu, nécessaire de rendre visible ce corps masqué par le pouvoir.

Les premières manifestations de l'A.F.D.D. ont lieu en 1977. Les familles des disparus descendent dans les rues munies de photographies de leurs proches et de cartons où chacun a porté une inscription particulière : " Où est mon fils ? ", " Où est mon père ? ", " Où est mon frère? ". Progressivement, ces cartons sont remplacés par des pancartes où est imprimée la photographie du disparu et qui portent cette unique inscription : " Où sont-ils? ". La quête d'un être cher devient alors véritablement quête d'un disparu. Les familles des victimes intègrent dans leur lutte pour la reconnaissance du crime, le fait que leurs proches ont été arrêtés parce qu'ils représentaient un danger pour le régime en place. La dimension politique du crime confère une dimension politique à leur quête menée, désormais, au nom de tous les disparus et aussi des idéaux que ceuxci pouvaient incarner ${ }^{15}$. Mais, la manifestation ne se veut pas qu'un espace de dénonciation. Les familles des victimes utilisent la seule trace de ce crime, la présence de l'absence, pour inscrire dans la mémoire de la société chilienne l'expérience de la disparition. Les pancartes révèlent des visages qui surgissent au milieu des passants comme autant de présences. Mais, ce sont les familles qui choisissent les moments où ces visages se laisseront voir. Certaines dates sont fixées en fonction de leur charge symbolique, pour commémorer la disparition des êtres chers ${ }^{16}$. La manifestation revêt alors le caractère d'un rite : le moment venu, les familles des disparus, défilent dans les rues de la ville, en suivant un parcours qui est le même chaque fois. C'est sur ce chemin 
qu'elles peuvent revendiquer la mémoire de leurs disparus, à défaut de pouvoir apprendre à vivre sans eux. Et ce sont ces images diverses (celles, immobiles, des pancartes et celles des femmes qui cherchent inlassablement) qui vont s'imposer à ceux qui ignoraient tout de la disparition, à ceux, aussi, qui préféraient ne pas savoir.

Les familles des disparus réinventent dans la rue un espace public, confisqué par le pouvoir militaire. Et, malgré les violences d'une police qui cherche systématiquement à les faire taire, leur message atteint la population chilienne parce qu'il n'est pas prisonnier de la parole. Il est intéressant de remarquer que c'est une danse qui deviendra la figure emblématique de la lutte des familles des disparus. Il s'agit d'une danse traditionnelle, et même, de la danse nationale par excellence : la cueca. En s'appropriant ce symbole de la nation, en l'associant au crime de la disparition-forcée, les familles s'attaquent à la légitimité de ce régime qui s'est attribué le droit exclusif de déterminer ce qui était bon ou mauvais pour la patrie. En s'appropriant cette danse, issue des traditions populaires, les familles se donnent aussi les moyens de communiquer à autrui ce qu'est la disparition vécue au quotidien. La cueca met en scène deux personnages typiques de la campagne chilienne : cette danse est le lieu de leur rencontre et de leur séduction. Sous les rythmes gais de l'accordéon et de la harpe, les partenaires se déplacent en suivant les contours imaginaires d'un " 8 " qu'ils ne peuvent pas quitter. L'homme traque la femme dans ce cercle : la danse est sa parade, sa mise en scène, son invitation. La femme se dérobe mais elle est celle qui poursuit autant qu'elle est poursuivie. Les personnages se contournent dans cet espace clos sans jamais se toucher. Pourtant, chacune de leur figure est une réponse à un geste, à un regard, à un sourire de l'autre. En 1978, les femmes des disparus créent la cueca sola. Cette danse conserve chaque accord et chaque déplacement de la cueca traditionnelle, mais les femmes sont seules dans le cercle. Quels gestes, quels regards, quels sourires leur indiquent-ils le chemin à suivre ? La silhouette des femmes, telle une ombre, ne fait que mieux signaler la présence impalpable, et pourtant " visible ", des hommes absents.

Présent de la disparition

14 Ces images hantent aujourd'hui le paysage politique chilien. Les femmes des disparus ont fait de leur quête une cause à part entière : en rendant visible le crime, elles viennent contredire le discours élaboré par les militaires mais surtout elles leur dénient le droit de déterminer les valeurs qui doivent être celles de la patrie. En effet, les militaires ont inscrit leur intervention du 11 septembre 1973, dans le cadre de la restauration de la démocratie, " bafouée " par le gouvernement socialiste. La disparition-forcée fut autant un effet qu'un moyen de cette stratégie qui cherchait à persuader tout un chacun de l'attachement des militaires aux valeurs de la démocratie. Et de fait, le conflit qui oppose les familles des disparus aux militaires est un conflit de valeurs. Ce qui est dénoncé, c'est certainement une pratique criminelle, mais c'est aussi cette usurpation du langage qui permit au régime militaire de se présenter comme étant le défenseur des libertés fondamentales : " Le Chili a toujours vécu dans le respect d'un ordre juridique qui est le reflet de l'attachement profond de chaque Chilien pour la dignité spirituelle de la personne humaine (...). C'est dans ce respect des droits de l'homme, que doit se trouver le ferment de la démocratie chilienne "17. Les images dévoilées par les femmes des disparus présentent à la population une autre vérité : les hommes et les femmes recherchés n'ont participé à aucune guerre, ils ne sont pas des ennemis de la patrie ni de la démocratie, mais des syndicalistes, des professionnels, des personnes nécessaires à la société, qui un jour ont été arrêtées par les autorités 
gouvernementales et ne sont jamais revenues ; leur absence doit être ressentie comme inacceptable par la société tout entière car elle est une atteinte à l'intégrité de la personne humaine, sans laquelle, effectivement, la démocratie ne serait qu'un vain mot.

Le changement de régime, advenu en 1989, n'a pas mis un terme à ce conflit. La manière dont s'est effectuée la transition démocratique, empêche de trancher une question qui heurte la sensibilité des militaires et des secteurs qui les ont soutenus ${ }^{18}$. Cependant, la thématique des violations des droits de l'homme offre au gouvernement démocratique le seul terrain sur lequel il puisse véritablement affirmer son autonomie à l'égard des Forces Armées. Il est donc de l'intérêt du gouvernement de ne pas étouffer ces questions mais de faire en sorte qu'elles trouvent une solution. Une des initiatives les plus importantes a été la création d'une commission d'enquête chargée de rendre compte des principaux crimes commis sous le régime militaire. Les familles des disparus sont à l'origine du projet dont l'un des objectifs principaux était de déterminer le sort subit par leurs proches. Le Rapport de la Commission Nationale de Vérité et de Réconciliation a été rendu public en mars 1992. Ce document assure la reconnaissance officielle des crimes commis et l'identification des victimes au cas par cas, mais il ne permet pas de retrouver le corps des disparus, ni de déterminer les circonstances de leur mort ${ }^{19}$. L'enquête menée par le gouvernement n'est pas une enquête judiciaire : sans la faculté de contraindre les individus impliqués, à témoigner, les faits ne peuvent être entièrement établis. Ainsi, si ce document s'adresse aux familles c'est moins pour éclaircir les crimes que pour mettre un terme à la déchirure profonde qui divise la population chilienne. Le Président Aylwin reconnaît que des crimes ont été commis et que la responsabilité morale incombe à la société en tant que telle et au nom de la Nation dont il est le représentant suprême, il demande pardon aux familles des victimes.

En l'absence de tout jugement, les familles des disparus rappellent à chacun, aujourd'hui comme hier, les images d'une nation salie par le crime. Ce message échappe aux voies traditionnelles de la prise de parole et ne serait être contenu. Les militaires le savent bien. C'est ce message irrespectueux qu'ils aimeraient pouvoir faire taire parce qu'il jette la suspicion sur un héritage qu'ils ont voulu glorieux : " Le 11 septembre nous fêtons la liberté du Chili. Les grossièretés lancées par ceux qui ont été vaincus et qui préparaient une guerre civile sont une offense. Ce sont eux les coupables. Ce sont eux les assassins "20. Il ne suffit pas de se savoir à l'abri de toute condamnation pénale, dès lors que les femmes qui dansent inscrivent, dans la mémoire de la population chilienne, le souvenir de ce crime dont chacun sait aujourd'hui qu'il fut l'œuvre des militaires. Les prochaines générations auront connaissance des pratiques criminelles du régime militaire car des messages officieux circulent dans la société chilienne et empruntent des voies diverses : celle des images révélées par les familles des victimes, celle de la parole des hommes politiques, celle de la culture aussi qui intègre progressivement ces images et ces paroles. La question n'est donc pas vraiment de savoir si ces crimes seront connus. Mais, de quelle manière seront-ils connus ? Le problème de l'impunité dont bénéficient les militaires aujourd'hui, porte peut-être moins sur la sanction des individus impliqués, que sur la sentence qui les déclarerait effectivement coupables ${ }^{21}$. Sans une parole officielle qui statue, non pas seulement sur les crimes mais encore sur la place de ces crimes dans la société chilienne, le discours 
des militaires continue à bénéficier d'une légitimité de fait. Il y a des silences qui sont comme des acquiescements ${ }^{22}$.

La réflexion sur les disparitions doit tenir compte de cette réalité faite à la fois de paroles et de silences, d'images visibles et d'images latentes. L'empreinte que laisse le crime dans la société chilienne n'est ni seulement le fait de la volonté de nuisance des auteurs, ni seulement le fait de la volonté de reconnaissance des familles. S'il existe une empreinte du crime c'est qu'elle émane de ce combat acharné qui cherche à transformer le crime en " expérience " qui puisse être léguée aux générations futures. Alors, de quoi parle-t-on lorsqu'on parle des disparitions ? Faut-il considérer que la disparition fut un excès de cette guerre que, selon les militaires, connaissait le pays en 1973 ? Faut-il considérer que la disparition fut un crime que, même en temps de guerre, la société ne saurait tolérer? Ces questions ne sauraient se réduire au seul problème de la disparition-forcée. Car, si ce crime pose véritablement un " problème ", c'est parce qu'il interroge la démocratie chilienne sur la nécessité de déterminer aujourd'hui ce que seront désormais les critères de l'Inacceptable.

\section{NOTES}

1.Ce référendum invitait la population chilienne à se prononcer sur la possibilité, pour le régime militaire, de rester au pouvoir huit années supplémentaires. A la grande surprise des autorités gouvernementales, le " non " l'emporta avec 54,71\% des suffrages contre $43,10 \%$ pour le " oui ". Ces résultats ont permis l'organisation d'élections présidentielles qui ont mis fin à dix-sept années de pouvoir militaire. 2.Une loi d'amnistie, votée en 1978, empêche de poursuivre en justice les militaires responsables de violations des droits de l'homme. Cette loi ne peut être abrogée en raison du système des " sénateurs désignés " (huit sénateurs ont été nommés par le général Pinochet) qui oblige le gouvernement à tenir compte des oppositions de la droite pinochetiste.

3.Cette coalition réunissait des forces politiques extrêmement hétérogènes. La participation de la Démocratie Chrétienne et du Parti Socialiste fut particulièrement problématique : en 1973, le leader démocrate-chrétien, Patricio Aylwin, avait refusé publiquement toute négociation avec le gouvernement de Salvador Allende. Ce passé a suscité des suspicions au sein des deux formations et provoqua de profondes divisions. Le rejet des crimes commis sous le régime militaire était le principal point de convergence des deux partis qui, en 1973, étaient adversaires.

4.Des manifestations massives ont lieu entre 1983 et 1986. Ce sont les travailleurs du cuivre qui sont à l'origine de ces " Journées de Protestation Nationale ", qui marquent le début d'une réorganisation du mouvement social au Chili.

5.Toute réflexion sur les " disparitions " suppose une interrogation première sur la nature même du phénomène. $C^{\prime}$ est là une entreprise particulièrement délicate car le terme recouvre des réalités diverses dont le seul dénominateur commun est l'" invisibilisation " des corps. Il est alors légitime de se demander si cette invisibilisation est une caractéristique suffisante pour attribuer à la disparition, le statut de " pratique 
coercitive ", ou encore, de " stratégie ". Ce problème conceptuel a fait l'objet d'un numéro de Cultures \& Conflits (" Disparitions ", n 13-14, printemps-été 1994). Notre réflexion prend appui sur un aspect de la question souligné notamment par l'analyse de Didier Bigo : l'invisibilisation des corps affecte à la fois un individu et son entourage. Et c'est cette capacité à agir sur une relation qui peut faire de la disparition un instrument aux mains du pouvoir.

6.Mais si la disparition peut être considérée comme un instrument du pouvoir, il faut néanmoins signaler que sa mise en œuvre est le fait de nombreux acteurs. C'est l'interaction entre ces acteurs, ayant des intérêts particuliers à défendre, qui permet véritablement la mise en place d'une pratique de la disparition-forcée, traversée par des logiques multiples. Dans le cas du Chili, c'est la D.I.N.A. (Direction de l'Intelligence Nationale) qui porte la responsabilité de la plupart des cas de disparitions-forcées. Sa création, en décembre 1973, témoigne de la volonté du gouvernement de rentabiliser les techniques de répression. En effet, la D.I.N.A. sera le seul service de renseignement dépendant directement du Général Pinochet. Ainsi, la disparition-forcée se présente comme étant un moyen de mieux cibler la répression tout en protégeant les exécutants de poursuites ultérieures. Par ailleurs, la D.I.N.A. est amenée à entrer en concurrence avec les autres services de renseignement, notamment dans le cadre de la répression du M.I.R. (Mouvement de Gauche Révolutionnaire). Dès lors, la disparition-forcée se présente aussi comme un moyen pour la D.I.N.A. de surpasser en efficacité les autres services.

7.Déclaration de la Junte. Journal officiel daté du 13 octobre 1973.

8.Les militaires peuvent d'autant plus facilement s'attribuer ce rôle, qu'ils se présentent à la population comme étant les héritiers d'une armée " libératrice " : celle qui, en 1818, a libéré le pays de l'autorité espagnole. En effet, les membres de la Junte légitiment leur intervention en soutenant la thèse de la guerre civile : le Chili était en guerre contre le marxisme-léninisme et c'est pourquoi, eux, militaires, étaient intervenus afin de libérer, une deuxième fois, le pays de ses ennemis.

9.Ces images sont fréquentes dans le discours des militaires. Ainsi, un décret édicté par la Junte en 1973, précise que " sur le nouveau gouvernement repose la mission d'extirper du Chili le marxisme-léninisme ", Extrait du décret-loi n 77 , cité dans le Rapport de la Commission Nationale de Vérité et Réconciliation, ibid., p. 100. 10.Les familles des victimes pouvaient faire une demande d'habeas corpus. Mais, l'occultation du corps en neutralisait les effets. Les tribunaux s'adressaient au Ministère de l'Intérieur où les autorités compétentes certifiaient que la personne recherchée n'avait jamais été arrêtée " par des agents dépendants dudit Ministère ". Ce respect des procédures habituelles était en complet décalage avec la réalité que vivait le pays : non seulement la police pouvait se rendre complice de ces arrestations mais surtout les disparitions-forcées étaient essentiellement le fait d'organisations secrètes. Le corps, soustrait aux yeux de la justice, ne pouvait ainsi être protégé : il n'y avait pas de corps et donc pas de prisonnier, pas de victime, pas de crime.

11.Cas de C. V. dont toute la famille a disparu. Interview réalisée à Santiago en septembre 1995.

12.Témoignage cité dans le Rapport de la Commission Nationale de Vérité et de Réconciliation, ibid., p. 176.

13.La disparition ne constitue pas véritablement " un " événement. Mais, elle suppose la succession de plusieurs événements mettant en jeu des acteurs divers. C'est la relation qui existe entre ces acteurs (le prisonnier, son entourage, les organismes de 
répression en concurrence les uns avec les autres) qui est au cœur du phénomène de disparition. Ainsi, " chercher la vérité ", c'est bien souvent se donner les moyens de remonter ces filières diverses. Cette dimension transforme la quête (du corps) en véritable enquête.

14.Cette réponse est parfois extrêmement explicite. Ainsi, le Ministre de l'Intérieur déclare solennellement, le 15 juin 1978, que : " le Gouvernement ne dispose pas d'antécédents pouvant prouver l'arrestation de ces personnes (...). Considérant que la plupart des supposés disparus sont, précisément, des activistes communistes (...), il est probable qu'ils sont morts dans des affrontements avec les forces de sécurité, sous de fausses identités (...) ". Cité dans le Rapport de la Commission Nationale de Vérité et de Réconciliation, p. 171.

15.La dimension politique coexiste toujours avec une dimension affective. C'est l'absence d'un être singulier (un fils, un époux, un père) qui est douloureuse. Cette singularité est présente dans la quête des familles et les visages sur les pancartes en sont une expression. Mais, pour devenir elles-mêmes des acteurs politiques, les familles doivent dépasser le caractère individuel du drame et révéler à la population chilienne, en quoi ce crime qui les affecte, constitue une blessure pour toute la société. La formulation au pluriel de l'interrogation qui figure sur les pancartes (" où sont-ils? ") témoigne de cette volonté car elle attire l'attention sur le fait que c'est une collectivité qui était visée : des hommes et des femmes susceptibles de constituer une opposition. La coexistence du politique et de l'affectif apparaît très nettement dans certains discours des membres de l'A.F.D.D. : " Nous les aimions pour ce qu'ils étaient. Nous les aimions parce qu'ils étaient libres dans leur manière de penser et justes dans leurs déterminations. Nous les aimions parce qu'ils étaient des dirigeants de partis politiques populaires, parce qu'ils étaient des dirigeants syndicaux, parce qu'ils étaient des professionnels motivés par le changement, par la vie. Les raisons qui nous ont conduit à les aimer ont été exactement celles que d'autres ont eu pour les faire disparaitre. A dixsept ans de ce crime de lèse-humanité, leur absence nous fait mal comme au premier jour ", Extrait du discours prononcé par Sola Sierra, présidente de l'A.F.D.D., à l'occasion de la cérémonie inaugurale de la 10e Semaine Internationale du DétenuDisparu en 1992.

16.Ce rituel de l'absence est établi en fonction de dates qui correspondent, tantôt à des événements particulièrement marquants dans la quête des familles (comme, par exemple, la publication des " listes des 119 "), tantôt à des événements qui acquièrent une valeur symbolique du fait même qu'ils sont choisis. Il en est ainsi de la Semaine Internationale du Détenu-Disparu, première initiative de la Fédération des familles des détenus-disparus en Amérique Latine (F.E.D.E.F.A.M.) qui coordonne l'action des familles au niveau du continent.

17." Déclaration de Principes du Gouvernement du Chili ", 11 mars 1974. Cité dans le Rapport de la Commission Nationale de Vérité et de Réconciliation, ibid. p. 14. 18.Les résultats du référendum montrent bien la profonde division de la population chilienne concernant les événements de 1973. Cette scission est une réalité avec laquelle le Gouvernement Aylwin a dû composer au jour le jour, elle permet de comprendre pourquoi le thème de la " réconciliation nationale " est devenu, dans le langage des autorités gouvernementales, un objectif politique à part entière. 19.Le statut de ce document est ambigu car il témoigne de la position du Gouvernement Aylwin mais constitue, en même temps, une source essentielle d'informations concernant la période 1973-1990. 
20.Propos tenus par le Général Pinochet, le 11 septembre 1995, à l'occasion de la commémoration du Coup d'État.

21.La récente condamnation du général Contreras pour l'assassinat de l'ex-ministre, Orlando Letelier, ne remet pas en cause cette impunité. Les circonstances particulières dans lesquelles ce crime a été exécuté lui donnent un caractère exceptionnel puisqu'il échappe à la loi d'amnistie : perpétré aux Etats-Unis, il coûta la vie à une citoyenne américaine, ce qui donna lieu à une pression constante des tribunaux nord-américains. Par ailleurs, il est possible de voir dans cette condamnation hautement symbolique (le général Contreras dirigea la D.I.N.A. de 1973 à 1977) la volonté de mettre un terme aux revendications des familles, puisque le " principal " responsable des disparitions est effectivement en prison. L'affaire Letelier est à l'origine d'une vaste polémique concernant l'interprétation adéquate de la loi d'amnistie qui constitue un obstacle essentiel à la poursuite judiciaire des militaires responsables. Le problème étant de savoir si cette loi annule le procès (comme c'est le cas actuellement) ou bien si elle doit s'appliquer une fois la sentence prononcée.

22.Seul le crime de la disparition est évoqué : les familles des disparus ont réussi à faire de leur quête une cause sans véritablement intégrer dans cette cause les autres crimes commis sous le régime militaire. Le débat politique tend alors à se refermer sur luimême. Résoudre le problème des violations des droits de l'homme cela veut dire pour les autorités gouvernementales, résoudre le problème des disparitions dans la mesure de ce " possible " que dictent les militaires.

\section{RÉSUMÉS}

Le crime de la disparition-forcée aura affecté plus d'une fois la société chilienne. C'est parce que l'occultation du corps de la victime produit également des effets sur son entourage que la disparition a pu devenir une pratique répressive aux mains des militaires. Hier, les familles des victimes transgressaient la loi du silence en rendant visible l'absence de leurs proches: leur révolte laisse une empreinte dans une société amenée à se prononcer aujourd'hui sur les crimes du passé et sur les normes qui doivent régir la démocratie.

More than once has the Chilean society been affected by forced disappearings. The effects produced by the occultation of the victim's body on the latter's family and friends have had such effects that the military have used the disappearing as a means of repression. The families previously transgressed the law of silence by making visible the absence of the close ones : their revolt left a mark in a society that today has to pronounce on the crimes of the past and the norms which are to rule democracy.

\section{INDEX}

Mots-clés : disparitions

Index géographique : Amérique du Sud, Chili 